\title{
Vascular smooth muscle function is associated with initiation and processing speed in patients with atherosclerotic vascular disease
}

\author{
DAVID J. MOSER, ${ }^{1}$ IVY N. MILLER, ${ }^{1}$ KARIN F. HOTH,${ }^{2,3}$ MARCELO CORREIA, ${ }^{4}$ \\ STEPHAN ARNDT, ${ }^{1}$ AND WILLIAM G. HAYNES ${ }^{4}$ \\ ${ }^{1}$ Department of Psychiatry, University of Iowa Carver College of Medicine, Iowa City, Iowa \\ ${ }^{2}$ Department of Medicine, National Jewish Medical and Research Center, Denver, Colorado \\ ${ }^{3}$ Department of Psychiatry, University of Colorado at Denver and Health Sciences Center, Denver, Colorado \\ ${ }^{4}$ Department of Internal Medicine, General Clinical Research Center, University of Iowa Carver College of Medicine, \\ Iowa City, Iowa
}

(Received January 4, 2007; Final Revision February 21, 2008; ACCEPTEd February 21, 2008)

\begin{abstract}
We previously reported a relationship between forearm resistance vessel function and global neuropsychological performance in patients with atherosclerotic vascular disease (AVD). This study was conducted to determine the relationships among vascular smooth muscle function, endothelial function, and initiation and processing speed in this sample. Participants were 80 individuals with AVD. Resistance vessel function was measured before and after infusion of vasoactive agents. Neuropsychological assessment included measures of estimated premorbid cognitive function, current global cognitive function, initiation, and processing speed. Vascular smooth muscle function was significantly associated with the initiation/processing speed composite score [R-Square Change $=.152 ; F$ Change $(1,71)=16.61 ; p<.001]$, above and beyond the variance accounted for by age, education, premorbid cognitive function, and endothelium-dependent vascular function. This relationship remained significant when controlling for current level of global cognitive functioning and 10 vascular risk factors. Endothelium-dependent vascular function was not significantly associated with test performance. Decreased vascular smooth muscle function in forearm resistance vessels was significantly associated with relatively poor initiation and processing speed in individuals with AVD. With additional research, measures of vascular function might become useful in the early identification of those individuals at greatest risk for vascular-related cognitive dysfunction. (JINS, 2008, 14, 535-541.)
\end{abstract}

Keywords: Vascular dementia, Subcortical vascular dementia, Neuropsychology, Atherosclerosis, Aging, Vascular endothelium-dependent relaxation

\section{INTRODUCTION}

It is known that vascular disease is a significant contributor to more than 50 percent of cases of dementia, regardless of specific dementia subtype (Breteler, 2000). Given that vascular disease and its associated effects on cognition are potentially modifiable entities (Bowler et al., 1999), it is important that we develop a better understanding of the pathophysiological mechanisms of this association. A nec-

Correspondence and reprint requests to: David J. Moser, Department of Psychiatry, W278 GH, University of Iowa Carver College of Medicine, 200 Hawkins Drive, Iowa City, IA 52242-1000. E-mail davidmoser@uiowa.edu essary component of this type of research is the accurate assessment of vascular disease severity, a process that can be quite challenging, given that vascular disease patients typically represent a very heterogeneous group with regard to the presence and severity of myriad vascular risk factors.

Forearm resistance vessel function, the ability of small forearm blood vessels to dilate when induced to do so using vasoactive agents, is a particularly intriguing physiological marker of vascular disease severity to study in relation to cognition. First, vascular function declines very early in the development of atherosclerotic vascular disease (AVD), before the appearance of atherosclerotic plaques (Lopez et al., 1989). Second, all known risk factors for 
AVD (hypertension, hyperlipidemia, obesity, and so on) are also associated with impaired vascular function (Vogel, 1999). Therefore, it is plausible that vascular function could serve as an integrated measure of total atherosclerotic risk factor burden, or a physiological proxy measure for overall vascular health. Finally, the methodology used in our research allows for the assessment of multiple aspects of vascular function, including vascular smooth muscle and endothelium-dependent vascular function. While it would be ideal to study the cerebral vasculature directly, it is not yet possible to conduct this type of detailed mechanistic analysis in the brain.

Although research on the relationships among forearm vascular function, cognition and cerebrovascular disease is a relatively new addition to the literature, recent studies suggest that this could lead to important findings regarding the mechanisms involved in these relationships. For example, Hoth et al. (2007) recently published findings suggesting a relationship between endothelium-dependent forearm vascular function and white matter hyperintensities on brain MRI. Additionally, we have recently published data demonstrating a significant and positive relationship between forearm resistance vessel function and global neuropsychological performance in individuals with atherosclerotic vascular disease (Moser et al., 2004, 2007). In our work, however, vascular smooth muscle function appeared to be more strongly associated with cognition than did endothelium-dependent function. These findings, within the context of those reported by Hoth et al., raise the possibility that various aspects of vascular function could affect the brain differentially. Additional research will be needed to determine whether this is the case.

In our previous work, neuropsychological function was measured using the Repeatable Battery for the Assessment of Neuropsychological Status (RBANS; Randolph, 1998). This test provides an efficient assessment of a range of cognitive abilities, although it is limited in some respects, including processing speed, initiation, and independent maintenance of behavior across time. These aspects of cognition are important because they are associated with level of daily functioning and are commonly impaired in vascular disease, more so than other abilities such as memory and language.

Using the same sample of participants with AVD that we included in our most recent study (Moser et al., 2007), we sought to determine the relationship between forearm resistance vessel function and performance on three measures of initiation and processing speed from our comprehensive neuropsychological battery. We hypothesized that vascular smooth muscle function, and not endotheliumdependent vascular function, would be significantly related to performance on tests of initiation and processing speed. The basis of this hypothesis was the fact that, as noted above, vascular smooth muscle function appeared to be particularly strongly associated with cognition in our previous work.

\section{METHODS}

This study was approved by the University of Iowa Institutional Review Board and signed informed consent was obtained from all participants. Research was completed in compliance with the Helsinki Declaration.

\section{Participants}

Eighty elderly individuals ( 34 women, 46 men; mean age = 68 years; $S D=7.8$; mean education $=14.6$ years; $S D=$ 3.2) were recruited from the University of Iowa Heart and Vascular Care Clinic and also from newspaper advertisements. All participants were age 55 or older, with a verified clinical diagnosis of AVD and a history of one or more of the following: angina pectoris, myocardial infarction, percutaneous transluminal coronary angioplasty, placement of coronary artery stent, and peripheral vascular disease (claudication). The numbers of participants taking medications were as follows: antihypertensives $=77(96 \%)$, statins $=$ $58(73 \%)$, nonsteroidal anti-inflammatories $=72(90 \%)$, angiotensin-converting enzyme inhibitors $=35(44 \%)$, Beta blockers $=60(75 \%)$, aspirin $=69(86 \%)$. Additionally, four women $(12 \%)$ were taking an estrogen supplement. Exclusion criteria included coronary artery bypass grafting, valve replacement, carotid endarterectomy, clinical history of large-vessel stroke, head injury with loss of consciousness longer than $30 \mathrm{~min}$, neurological disorder or systemic illness unrelated to vascular disease that is likely to affect cognition, focal neurological sign, current or past severe psychiatric illness (e.g., bipolar affective disorder, schizophrenia), and clinical diagnosis of dementia. All subjects were determined, by means of interview, to have intact decisional capacity for informed consent and were independent in daily functioning. All subjects underwent thorough history and physical examinations conducted by a physician, including neurological exam, electrocardiogram, and fasting blood draw/analysis.

\section{Forearm Resistance Vessel Function Assessment}

Beginning the night before testing, subjects fasted and refrained from taking any medication until all vascular study procedures were completed the following morning. Forearm blood flow was measured in both arms before and after infusion of vasoactive agents (into one arm) using venous occlusion plethysmography with mercury-in-Silastic strain gauges. This technique is currently the gold standard for measuring resistance vessel function in the forearm, requires virtually no subjective judgment on the part of the technician, and has been shown to yield highly reproducible results in our laboratory. During this procedure, the left brachial artery was cannulated under local anesthesia with a 27 -gauge steel needle attached to an 18-gauge epidural catheter. Baseline forearm blood flows were obtained during infusion of $0.9 \%$ saline $(1 \mathrm{ml} / \mathrm{min})$ for $30 \mathrm{~min}$. Acetylcholine $(3-30 \mu \mathrm{g} /$ 
min), nitroprusside (1-10 $\mu \mathrm{g} / \mathrm{min})$, and verapamil (10$100 \mu \mathrm{g} / \mathrm{min}$ ) were then infused separately into the left arm, with each dose infused for $6 \mathrm{~min}$. The order of acetylcholine and nitroprusside was randomized, but verapamil was always administered last due to its longer duration of action.

These three vasoactive agents were administered to examine specific aspects of vascular function. Intra-arterial acetylcholine causes vasodilation through stimulation of endothelial cell muscarinic receptors, thereby activating endothelial nitric oxide synthase and increasing nitric oxide production, which then relaxes underlying vascular smooth muscle, resulting in resistance vessel dilatation. Nitroprusside, on the other hand, is a direct nitric oxide donor, causing vascular smooth muscle relaxation without involvement of endothelial cells. Verapamil acts directly upon vascular smooth muscle cells, causing relaxation without altering endothelial cell function or generation of nitric oxide. Based on the different mechanisms of these agents, vasodilation that occurs as the result of acetylcholine administration is often referred to as "endothelium-dependent" and that which occurs in response to nitroprusside and verapamil is described as "endothelium-independent." It should be noted, however, that examining response to acetylcholine alone does not yield a truly specific index of endothelium-dependent vasodilation. For example, even if vascular smooth muscle dysfunction occurred alone, vasodilation in response to acetylcholine would still be impaired, despite the fact that endothelial function would be normal. Therefore, the most robust parameter for endotheliumdependent vasodilation is obtained by assessing vascular response to acetylcholine and correcting that for dilatation that occurs in response to a vascular smooth muscle specific dilator. In this study, we chose to accomplish this by using a simple acetylcholine response/verapamil response ratio.

With each drug, forearm blood flow was measured during the last $3 \mathrm{~min}$ of each 6-min dose. Saline was infused for at least $12 \mathrm{~min}$ between drugs to allow blood flow to return to basal levels. Blood flows were measured in the noninfused (control) arm at time points identical to those in the infused arm so as to provide a contemporaneous control for random fluctuations in flow.

The outcome measure for each drug was the mean percent increase in forearm blood flow in the infused arm following infusion of the three doses of that drug, relative to baseline, adjusted for changes in blood flow in the noninfused arm. Higher values indicated better resistance vessel function. Neuropsychological assessment and blood vessel testing were conducted by separate individuals, each of whom was blind to the other's data.

\section{Neuropsychological Assessment}

Participants underwent a comprehensive neuropsychological assessment, from which the following three measures were selected for analysis in this study: Controlled Oral Word Association (Benton \& Hamsher, 1989); Wechsler
Adult Intelligence Scale-III Digit Symbol-Coding (Wechsler, 1997); Trail Making Test-Part A (Reitan, 1958). Additionally, Wide Range Achievement Test-3 (WRAT-3) Reading (Wilkinson \& Webb, 2001) score and RBANS Total Scale Score (Randolph, 1998) were used to control for premorbid cognitive functioning and global cognitive functioning, respectively, as described below. All tests were administered and scored by a trained research assistant who was blind to vascular assessment data.

\section{Statistical Analysis}

Raw scores on the three outcome measures were standardized using $z$-score transformation, based on the means and standard deviation of each variable from this sample. All scores were calculated such that higher values indicated better performance. A composite score was then calculated for each participant by averaging his/her $z$-scores across all three measures.

A hierarchical multiple regression model, with the initiation/processing speed composite score as the dependent variable, was used to test our main hypothesis. Three blocks of independent variables were entered in succession, as follows: Block 1-age, education, estimated level of premorbid cognitive function (WRAT-3 Reading score); Block 2-endothelium-dependent vascular function (acetylcholine response/verapamil response ratio); Block 3response to verapamil was entered as a third block. Follow-up partial correlations were then conducted to determine the relationships between measures of vascular function and performance on the three individual tests that made up the initiation/processing speed composite score.

To determine the possible effects of more conventionally studied vascular risk factors and related variables on the outcome of the above analyses, we sought to determine whether such variables shared similar relationships with both vascular smooth muscle function and endotheliumdependent vascular function. This was accomplished using two regression models, one with response to verapamil as the dependent variable and one with the acetylcholine response/verapamil response ratio as the dependent variable. The same block of independent variables was used in each model, as follows: age, body mass index, systolic blood pressure, diastolic blood pressure, total cholesterol, LDL-C, HDL-C, triglycerides, homocysteine, glucose, and C-reactive protein.

Once it had been determined that vascular smooth muscle function was, indeed, significantly related to initiation/ processing speed (controlling for age, education, and estimated level of premorbid cognitive function), we sought to determine whether this relationship existed above and beyond the relationship between vascular smooth muscle and global cognitive function that we have reported previously. This was determined by calculating a partial correlation between response to verapamil and initiation/processing speed composite score, controlling for age, education, and RBANS Total Scale Score. Finally, to determine whether the 


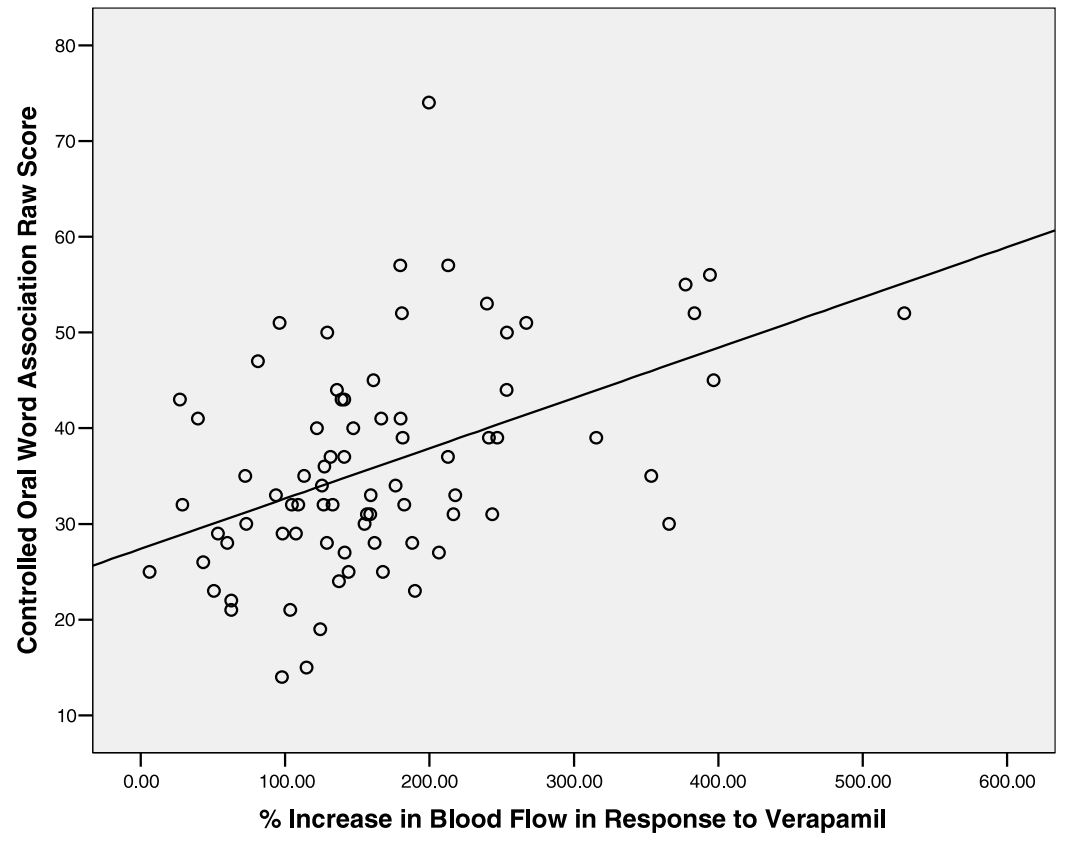

Fig. 1. Relationship between forearm vascular smooth muscle function and Controlled Oral Word Association Performance.

relationship between vascular smooth muscle function and initiation/processing speed existed above and beyond the effects of more conventional vascular risk factors, a partial correlation was calculated between response to verapamil and the initiation/processing speed composite score, controlling for age, education, WRAT-3 Reading score, and the 10 vascular risk factors and related variables described above.

\section{RESULTS}

Descriptive statistics are shown in Tables 1 and 2. With regard to neuropsychological test performance, group Means for all tests fell within normal limits relative to appropriate norms.

Hierarchical multiple regression revealed that age, education, and estimated level of premorbid cognitive function were significantly associated with the initiation/processing speed composite score $\left[R^{2}=.187 ; F(3,73)=5.59 ; p=\right.$ $.002]$. Then, the addition of endothelium-dependent vascular function to the model showed that this variable did not account for significant additional variance in the composite score $\left[R^{2}\right.$ Change $=.013 ; F$ Change $\left.(1,72) ; p=.288\right]$. Finally, the addition of vascular smooth muscle function to the model revealed that this variable was significantly associated with the composite score, above and beyond the variance already accounted for by the variables previously entered into the model $\left[R^{2}\right.$ Change $=.152 ; F$ Change $(1,71)=$ $16.61 ; p<.001]$.

Follow-up partial correlations revealed that, after controlling for age, education, and estimated level of premorbid cognitive function, vascular smooth muscle function was significantly related to Controlled Oral Word Associa- tion performance (partial $r=.439 ; p<.001$ ) and Digit Symbol-Coding (partial $r=.237 ; p=.042$ ) performance. Endothelium-dependent vascular function was not significantly related to performance on any of the three tests included in the initiation/processing speed component. See Table 3.

The final two regression models revealed that the 10 vascular risk factors and related variables included in the study (see above for a list) accounted for similar amounts of variance in both vascular smooth muscle function $\left[R^{2}=.237\right.$;

Table 1. Vascular function scores and related variables

\begin{tabular}{lrr}
\hline \hline & Mean & $S D$ \\
\hline$\% \Delta$ in blood flow with acetylcholine & 152 & 104 \\
$\% \Delta$ in blood flow with verapamil & 167 & 98 \\
Body mass index & 31 & 7 \\
Systolic blood pressure, mm Hg & 135 & 21 \\
Diastolic blood pressure, mm Hg & 72 & 11 \\
Total cholesterol, mg/dl & 169 & 40 \\
HDL-C, mg/dl & 51 & 16 \\
LDL-C, mg/dl & 89 & 29 \\
Glucose, mg/dl & 116 & 32 \\
Triglycerides, mg/dl & 143 & 71 \\
Homocysteine, $\mu \mathrm{M} / \mathrm{L}$ & 11.98 & 4.26 \\
C-Reactive Protein, mg/L & 4.35 & 4.64
\end{tabular}

Note. $N=80$, except for homocysteine $(N=73)$, LDL-C $(N=79)$, and C-Reactive Protein $(N=75)$. Blood flow $\Delta$ is the percent increase in forearm blood flow in response to intra-arterial infusion of drug, adjusted for changes in blood flow in the noninfused arm (higher values indicate better vascular function); LDL-C $=$ low-density-lipoprotein cholesterol; HDL-C $=$ high-density-lipoprotein cholesterol. 
Table 2. Neuropsychological test scores

\begin{tabular}{lcc}
\hline \hline & Mean & $S D$ \\
\hline Controlled Oral Word Association & 35.74 & 11.22 \\
& $(\mathrm{~T}=50)$ & \\
Digit Symbol-Coding & 54.74 & 12.08 \\
& $(\mathrm{~T}=51)$ & \\
Trail Making Test-A & 35.84 & 12.04 \\
& $(\mathrm{~T}=50)$ & \\
Wide Range Achievement Test-3 & 101.41 & 7.85 \\
$\quad$ Word Reading Standard Score & $(\mathrm{T}=51)$ & \\
RBANS Total Scale Score & 97.08 & 11.46 \\
& $(\mathrm{~T}=48)$ & \\
&
\end{tabular}

Note. $N=80$. Controlled Oral Word Association, Digit Symbol-Coding, and Trail Making Test-A values are raw scores. Wide Range Achievement Test-3 and RBANS (Repeatable Battery for the Assessment of Neuropsychological Status) values are Standard Scores (normative Mean $=100$; $S D=15)$. Values in parentheses are $\mathrm{T}$ scores relative to published agecorrected norms (normative Mean $=50 ; S D=10$; Ivnik et al., 1996; Wechsler, 1997).

$F(11,56)=1.581 ; p=.130]$ and in endothelium-dependent vascular function $\left[R^{2}=.189 ; F(11,56)=1.181 ; p=.317\right]$, with neither model reaching statistical significance.

The partial correlations described above revealed a significant relationship between vascular smooth muscle function and initiation/processing speed when controlling for age, education, and global cognitive function (RBANS Total Scale Score), with partial $r=.30 ; p=.009$. The relationship between vascular smooth muscle function and initiation/ processing speed also remained significant when controlling for age, education, premorbid cognitive function (WRAT-3 Reading score), and the 10 vascular risk factors and related variables described above ( artial $r=.28 ; p=.039$ ).

\section{DISCUSSION}

These findings support our hypothesis and indicate that forearm vascular smooth muscle function is significantly asso-

Table 3. Relationships among vascular smooth muscle function, endothelium-dependent vascular function, and initiation/ processing speed

\begin{tabular}{lcc}
\hline \hline & $\begin{array}{c}\% \Delta \text { in } \\
\text { blood flow } \\
\text { with verapamil }\end{array}$ & $\begin{array}{c}\text { Acetylcholine/ } \\
\text { verapamil } \\
\text { response ratio }\end{array}$ \\
\hline $\begin{array}{c}\text { Initiation/Processing } \\
\text { Speed Composite }\end{array}$ & $.371^{*}$ & .125 \\
$\begin{array}{c}\text { Controlled Oral } \\
\text { Word Association }\end{array}$ & $.439 * *$ & .040 \\
$\begin{array}{c}\text { Digit Symbol-Coding } \\
\text { Trail Making Test-A }\end{array}$ & $.237 *$ & .189 \\
\hline
\end{tabular}

Note. Values are partial correlations, controlling for age, education, and estimated level of premorbid cognitive function (Wide Range Achievement Test-3 Reading score). $* \mathrm{p}<.05, * * \mathrm{p}<.001$; Correlations were calculated such that positive correlations indicate that better vascular function is associated with better test performance. ciated with performance on tests of initiation and processing speed, whereas endothelium-dependent vascular function was not associated with these measures. Of note, the relationship between vascular smooth muscle function and test performance was shown to exist above and beyond the effects of age, education, estimated level of premorbid cognitive function, current global cognitive function, and 10 conventionally studied vascular risk factors (blood pressure, lipid levels, and so on). Follow-up analyses revealed that vascular smooth muscle function was most strongly associated with performance on Controlled Oral Word Association (Benton \& Hamsher, 1989) and Digit Symbol-Coding (Wechsler, 1997), tests that place demand on initiation, speed, and independent maintenance of effort across time.

Several factors must be considered in weighing the importance of these findings. First, it is important to consider that the participants in this study were relatively healthy compared with the larger existing population of individuals with vascular disease. Specifically, they were all receiving close management of their illness, they had not undergone cardiac surgery, had not shown clinical evidence of large vessel stroke, and did not meet criteria for dementia. Furthermore, as shown in Tables 1 and 2, their scores on various vascular-related and neuropsychological variables indicate that, as a group, they were not severely ill or demonstrating significant cognitive impairment, although there were a wide range of scores across these variables. Therefore, it is intriguing that a significant relationship between resistance vessel function, initiation and processing speed would already be evident in these individuals, even at this relatively early disease stage. It is quite plausible that this relationship will grow stronger with the worsening of both vascular disease and cognitive performance, although we do not yet have sufficient longitudinal data to determine whether this is the case.

Second, problems with initiation and psychomotor slowing are among the symptoms most commonly seen in patients with early-stage vascular cognitive decline (de Groot et al., 2000; Inzitari et al., 2000; Looi \& Sachdev, 2000). Although such symptoms might be mistaken for the relatively benign effects of normal aging (Royall et al., 2000), they are commonly due to vascular disease and can have profound effects on basic daily functioning and quality of life (Boyle \& Cahn-Weiner, 2005; Boyle et al., 2002). This study supports the notion that these abilities are particularly susceptible to early-stage vascular disease and raises the possibility that, with additional longitudinal research, measures of vascular function could become useful in predicting which individuals are at greatest risk for continued decline in these abilities and other aspects of cognitive function.

In this study, measures of forearm resistance vessel function were used not only as proxy indicators of overall vascular health, but also to tease apart the specific aspects of vascular dysfunction that are most closely associated with cognitive ability. Forearm measures allow for such specific study through the infusion of various vasoactive agents, as described above, which would not be practical 
in the cerebral vasculature. Given that atherosclerosis is known to be a generalized process (Lie, 1994), it is plausible that individuals with vessel dysfunction in the periphery may also have early-stage cerebrovascular pathology (e.g., small vessel disease), which is known to be associated with cognitive deficits such as slowing and attentional dysfunction.

As noted above, Hoth et al. (2007) recently published data showing a significant relationship between endothelial function, measured in the brachial artery, and volume of white matter hyperintensities in a sample of older adults with cardiovascular disease. It might be expected, therefore, that the current study would have shown a stronger relationship between endothelium-dependent function and cognitive performance, yet this was not the case. Our findings suggest that vascular smooth muscle function is much more strongly associated with cognition, raising the possibility that this relationship exists independently of the relationship between endothelial function and brain structure. Additionally, it should be considered that the measures of vascular function used in the current study are positively correlated with, but quite different from (Irace et al., 2001) those used by Hoth et al. Specifically, the current study involved assessment of resistance vessels, as opposed to the brachial artery, and the two are physiologically different from one another with regard to the manner in which they regulate blood flow and their susceptibility to various risk factors. Additionally, although the brachial artery ultrasound method is widely used, its measure of endotheliumindependent vessel function involves administration of a single, high dose of nitroglycerin. For reasons discussed by (Raitakari et al., 2001), this method cannot definitively detect endothelium-independent dysfunction (e.g., vascular smooth muscle dysfunction). Therefore, while each of the above methodologies provides important information regarding vascular function and its relationship to brain function, additional research will be necessary to better define the specific mechanisms involved in this relationship.

There are limitations of the current study that should be discussed. First, as noted above, participants were recruited primarily from a cardiology clinic and had well-managed disease, raising the possibility that the above findings may not generalize across all individuals with vascular disease. Furthermore, it was not possible to determine the effects of medication status on our findings, as virtually all participants were taking multiple vascular-related medications, which could not be withheld for any extended period of time due to obvious ethical considerations. Although participants refrained from taking medications starting the night before vascular testing, the effects of these various agents were certainly not entirely controlled by this methodology. Unfortunately, this problem is currently unavoidable and is common across studies of vascular disease and cognition. It is conceivable that medication effects caused artificial normalization of the risk factor variables included in this study, and this could have affected the degree to which vascular smooth muscle response was shown to be associated with cognitive performance above and beyond the effects of these risk factors. Conversely, however, it must also be considered that vascular disease medications (e.g., lipid lowering agents) are known to improve vascular function. Therefore, it could be expected that, while such medications may have weakened the relationships between conventional vascular risk factors and cognition in our sample, the relationship between vascular function and cognition should have been similarly attenuated, yet this association remained significant.

It is also important to note that the various conventional risk factors that were studied shared similar relationships with both endothelium-dependent and vascular smooth muscle function. Therefore, the finding that vascular smooth muscle function was more strongly associated with cognitive performance is not likely confounded by a differential effect of conventional vascular risk factors on our vascular measures. Finally, it must be noted that this study lacked a healthy comparison group and, therefore, it cannot be determined whether or not the relationship found between vascular function and neuropsychological test performance is specific to individuals with AVD or might apply to the elderly population in general, as part of the normal aging process.

In summary, our findings demonstrate a significant and positive relationship between forearm vascular smooth muscle function and initiation and processing speed in a sample of elderly patients with relatively early-stage atherosclerotic vascular disease. These findings augment existing data that have shown a relationship between vascular function and global neuropsychological performance in such patients Moser et al., 2004, 2007). Additional research will be necessary to determine more specifically the mechanism involved in this relationship. With additional study and improvements in vascular assessment, measures of vascular function may ultimately become increasingly useful in identifying the mechanisms by which vascular disease causes cognitive dysfunction and how to prevent or attenuate this process.

\section{ACKNOWLEDGMENTS}

This research was supported by grants to Dr. David J. Moser from the National Institute on Aging (1 K23 AG020649-01A1), the American Federation for Aging Research, and the University of Iowa. Additional support was provided by the University of Iowa General Clinical Research Center (Grant RR00059 from the General Clinical Research Centers Program, National Center for Research Resources, NIH). The authors thank Stephanie M. Hynes, B.S., Becky Reese, B.S., and Clare Humphreys, B.A. for their assistance with subject recruitment and data collection.

\section{REFERENCES}

Benton, A.L. \& Hamsher, K. (1989). Multilingual Aphasia Examination (2nd ed.). Iowa City, IA: AJA Associates.

Bowler, J.V., Steenhuis, R., \& Hachinski, V. (1999). Conceptual background to vascular cognitive impairment. Alzheimer Disease and Associated Disorders, 13(Suppl. 3), S30-S37. 
Boyle, P.A. \& Cahn-Weiner, D. (2005). Functional impairment in vascular dementia. In R. Paul, R. Cohen, B.R. Ott, \& S. Salloway (Eds.), Vascular Dementia. Totowa, NJ: Humana Press.

Boyle, P.A., Cohen, R.A., Paul, R., Moser, D., \& Gordon, N. (2002). Cognitive and motor impairments predict functional declines in patients with vascular dementia. International Journal of Geriatric Psychiatry, 17, 164-169.

Breteler, M.M. (2000). Vascular risk factors for Alzheimer's disease: An epidemiologic perspective. Neurobiology of Aging, 21, 153-160.

de Groot, J.C., de Leeuw, F.E., Oudkerk, M., van Gijn, J., Hofman, A., Jolles, J., \& Breteler, M.M. (2000). Cerebral white matter lesions and cognitive function: The Rotterdam Scan Study. Annals of Neurology, 47, 145-151.

Hoth, K.F., Tate, D.F., Poppas, A., Forman, D.E., Gunstad, J., Moser, D.J., Paul, R.H., Jefferson, A.L., Haley, A.P., \& Cohen, R.A. (2007). Endothelial function and white matter hyperintensities in older adults with cardiovascular disease. Stroke, 38, 308-312.

Inzitari, D., Erkinjuntti, T., Wallin, A., Del Ser, T., Romanelli, M., \& Pantoni, L. (2000). Subcortical vascular dementia as a specific target for clinical trials. Annals of the New York Academy of Science, 903, 510-521.

Irace, C., Ceravolo, R., Notarangelo, L., Crescenzo, A., Ventura, G., Tamburrini, O., Perticone, F., \& Gnasso, A. (2001). Comparison of endothelial function evaluated by strain gauge plethysmography and brachial artery ultrasound. Atherosclerosis, $158,53-59$.

Ivnik, R.J., Malec, J.F., Smith, G.E., Tangalos, E.G., \& Petersen, R.C. (1996). Neuropsychological Tests' Norms Above Age 55: COWAT, BNT, MAE Token, WRAT-R Reading, AMNART, STROOP, TMT, and JLO. The Clinical Neuropsychologist, 10, 262-278.

Lie, J.T. (1994). Pathology of occlusive disease in the extracranial arteries. In F.B. Meyer (Ed.), Sundt's Occlusive Cerebrovascular Disease (pp. 25-44). Philadelphia: W.B. Saunders Co.

Looi, J.C. \& Sachdev, P.S. (2000). Vascular dementia as a frontal subcortical system dysfunction. Psychological Medicine, 30, 997-1003.
Lopez, J.A., Armstrong, M.L., Piegors, D.J., \& Heistad, D.D. (1989). Effect of early and advanced atherosclerosis on vascular responses to serotonin, thromboxane A2, and ADP. Circulation, 79, 698-705.

Moser, D.J., Hoth, K.F., Robinson, R.G., Paulsen, J.S., Sinkey, C.A., Benjamin, M.L., Schultz, S.K., \& Haynes, W.G. (2004). Blood vessel function and cognition in elderly patients with atherosclerosis. Stroke, 35, e369-e372.

Moser, D.J., Robinson, R.G., Hynes, S.M., Reese, R.L., Arndt, S., Paulsen, J.S., \& Haynes, W.G. (2007). Neuropsychological performance is associated with vascular function in patients with atherosclerotic vascular disease. Arteriosclerosis Thrombosis and Vascular Biology, 27, 141-146.

Raitakari, O.T., Seale, J.P., \& Celermajer, D.S. (2001). Impaired vascular responses to nitroglycerin in subjects with coronary atherosclerosis. American Journal of Cardiology, 87, 217219, A218.

Randolph, C. (1998). Repeatable Battery for the Assessment of Neuropsychological Status. San Antonio, TX: The Psychological Corporation.

Reitan, R.M. (1958). Validity of the Trail Making Test as an indicator of organic brain damage. Perceptual and Motor Skills, 8, 271-276.

Royall, D.R., Chiodo, L.K., \& Polk, M.J. (2000). Correlates of disability among elderly retirees with "subclinical" cognitive impairment. The Journals of Gerontology. Series A. Biological Sciences and Medical Sciences, 55, M541-M546.

Vogel, R.A. (1999). Brachial artery ultrasound: A noninvasive tool in the assessment of triglyceride-rich lipoproteins. Clinical Cardiology, 22(6 Suppl), II34-39.

Wechsler, D. (1997). Wechsler Adult Intelligence Scale-Third Edition. San Antonio, TX: The Psychological Corporation.

Wilkinson, I.B. \& Webb, D.J. (2001). Venous occlusion plethysmography in cardiovascular research: Methodology and clinical applications. British Journal of Clinical Pharmacology, 52, 631-646. 